\title{
Optical transport network layered architecture for the MOONET
}

Slobodanka Tomic, Siemens AG Austria

Correspondence address:

Slobodanka Tomic, Siemens AG Austria, Department PSE EZE TNT4, Erdbergerlaende 26, A-1030 Vienna, Austria

\begin{abstract}
The layered network modelling, as defined in ITU G.805 [1], provides a means to describe, in a uniform way, the information transfer capability of the different transport networks, as well as, to deal with the inter-working and the management interoperability between the different transport networks. It provides a means to describe the network functionality in an abstract way in terms of a small number of architectural components.

The layering and partitioning concepts are used for the definition of the transport network layered architectures of the existing SDH network in G.803, and for the SDH-based ATM network defined in I.311. They are also applied to the optical transport networks. The standardisation of the layered architecture model for the optical networks described in ITU G.otn [2] is currently in progress, and some other models [4], [6] are also introduced. So far, the architecture proposed deals with the optical path network [6], which is characterised by the combination of WDM transmission and the wavelength routing.
\end{abstract}

The management of the optical path network is also in the scope of the project MOON (Management of Optical Networks). MOON is one of the EC's ACTS projects with a task to establish a conceptual TMN framework for the management of all-optical WDM transport networks and to demon-

This work was supported in part by the European Commission. The contents of this paper is solely the responsibility of the authors. 
strate the developed concepts and the feasibility of the adopted approach in a field trial network MOONET.

One of the main objectives of the project MOON is to elaborate the generic information model for all-optical WDM transport networks. For this task, the definition of the layered architecture model for the optical transport network is considered to be a starting point.

The layered architecture model covers all the technology aspects of the optical transport network. It can be used for equipment modelling, network access modelling, and the network resource modelling. For each layer network a managed process which interacts with its counter-part in the same layer or with the adjacent layer management processes can be defined. This process can be partly assigned to the adaptation and termination functions of the layer network. It must also provide an interface to the TMN agent. In this way the interaction of the OAM and the TMN can be modelled. The interaction between two different technology transport networks can be modelled with the interaction between management processes of two adjacent layer networks which belong to different technology transport networks.

In the project MOON the approach similar to the approach utilised in [5] is taken for the definition of the management information model. This comprises the utilisation of the adopted layered network architecture in modelling of the network elements and all-optical equipment, the identification of the network resources, and the definition of the related management capabilities.

The emerging management information model would be demonstrated in the field trial network MOONET. Due to the relatively simple topology of the MOONET not all of the concepts established in MOON could be verified. Still, the activities in the project MOON are aimed to give the contribution to the general view of the optical network modelling and management.

\section{Introduction}

The layered network modelling, as defined in ITU G.805 [1], provides a means to describe, in a uniform way, the information transfer capability of the different transport networks, as well as, to deal with the inter-working and the management interoperability between the different transport net- 
works. It provides a means to describe the network functionality in an abstract way in terms of a small number of architectural components.

From the telecommunications management point of view, the layering and partitioning concepts as defined in the G.805 support transport network structuring into independent manageable parts.

The optical transport network can be decomposed into a number of independent layer networks, with a client/server relationship between adjacent layer networks.

The layering concept provide a means for the independent design and operation of each layer, but with the similar functions. This gives the possibility to encapsulate in each layer its own operations, diagnostic and automatic failure recovery, but still have the similar "look-and-feel" in the management and operation of all layers.

On the other hand each layer network is uniquely identified with its information transfer capability, i.e. the input characteristic information it can adapt , which is relevant for the server role of the layer network, and its own, output, characteristic information related to its client role. So, the layering approach provides also, that the adding or modifying of a layer network can be done without affecting other layers from architectural viewpoint, provided that the characteristic information definition at its input and output is preserved. This should lead to the simple modelling of networks that contain multiple transport technologies.

The partitioning concept as defined in the G.805 is applied in order to define the network structure and domain or administrative boundaries in the network.

The topology of each layer network - the network structure, can be described by a means of the topological components, and the reference points[1]. The transparent information transfer across a topological component can be represented by a means of related transport entities[1]. In the sense of the network management these components represent network resources.

The layering and partitioning concepts are used for the definition of the transport network layered architectures of the existing SDH network in G.803, and for the SDH-based ATM network defined in 1.311. They are also applied to the optical transport networks. The standardisation of the layered architecture model for the optical networks described in ITU G.otn [2] is currently in progress, and some other models [4], [6] are also introduced. So far, the architecture proposed deals with the optical path network [6], 
which is characterised by the combination of WDM transmission and the wavelength routing.

The management of the optical path network is also in the scope of the project MOON (Management of Optical Networks). MOON is one of the EC's ACTS projects with a task to establish a conceptual TMN framework for the management of all-optical WDM transport networks and to demonstrate the developed concepts and the feasibility of the adopted approach in a field trial network MOONET.

The field trial network, MOONET, will be a meshed network, with the alloptical core consisting of three optical cross-connects. From the aspect of the network element management, the management of the optical crossconnects and the optical line amplifiers included in one of the links will be demonstrated. No wavelength routing is supported in the network.

In the Figure 2-1 MOONET, the field trail network of the project MOON, is shown. The core network comprises three OXC located at the locations Passau and Schaerding. The MOONET represents both in respect of equipment and topology the extended field trial network of the project PHOTON.

One of the main objectives of the project MOON is to elaborate the generic information model for all-optical WDM transport networks. The management information model transforms the transport oriented network view concerned with network layers, optical paths, connections and trails, where the access- and termination-points and the adaptation functions are located in the network components and the equipment oriented network view concerned with network components such as terminal multiplexers, cross connects, add and drop multiplexers, and amplifiers, connected by fibre links carrying either single channel signals (SCS) supported by a lightwave with a distinct frequency, or multi channel signals (MCS), where each channel is supported by a lightwave of a different frequency, into a management view of a network, concerned with the managed objects which represent network resources.

For this task, the definition of the layered architecture model for the optical transport network is considered to be a starting point. 


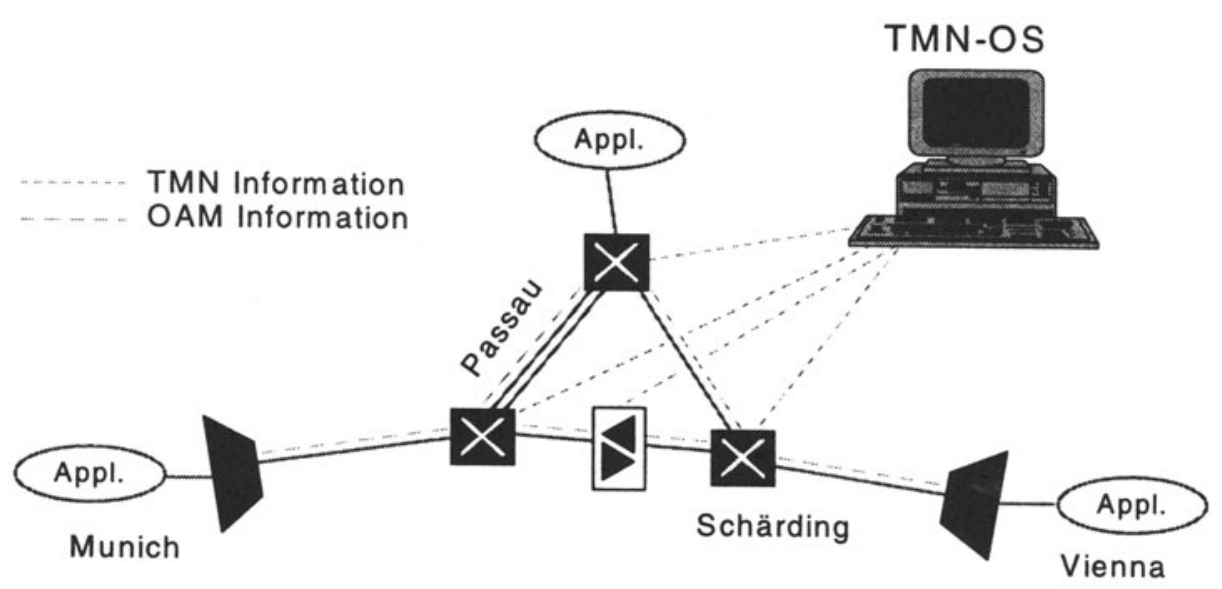

Fig. 1-1 MOONET 


\section{Technical approach}

The architecture model for the optical network must provide answers for the set of questions such as:

- Where is the boundary of the optical network, what is the logical and physical content of the characteristic information on this boundary and of the characteristic information of each layer network,

- What is the transport and management content of the client/server relationship between layers, what is the "service" offered by each optical layer network and how this service is supported and guaranteed by a „protocol". specific for this layer network. This includes also the definition of the logical and physical content of the overhead information.

For the definition of the boundary of the optical network the model described in [7] and depicted in Fig. 2-1 is used. The model is enhanced with the optical inter-network adapter which should depict that possibly different optical networks could co-exist and should be able to interwork.

In the Fig. 2-1 the user of the optical network interact with the optical network through the optical network adapter. The optical network adapter provides the user with the transparent access to the networking capabilities of the optical network, and transforms the arbitrary user characteristic information in the optical signal, which can undergo all possible transformations in the all-optical network, such as frequency conversion, dispersion accommodation, regeneration, and still carry unmodified user information. Both of this aspects should find its representation in the transport network architecture model.

Inside the optical network only all-optical data paths exist with defined, and in the future standardised, logical and physical content. Two different optical networks which support different optical paths can inter-work through optical inter-network adapter . 


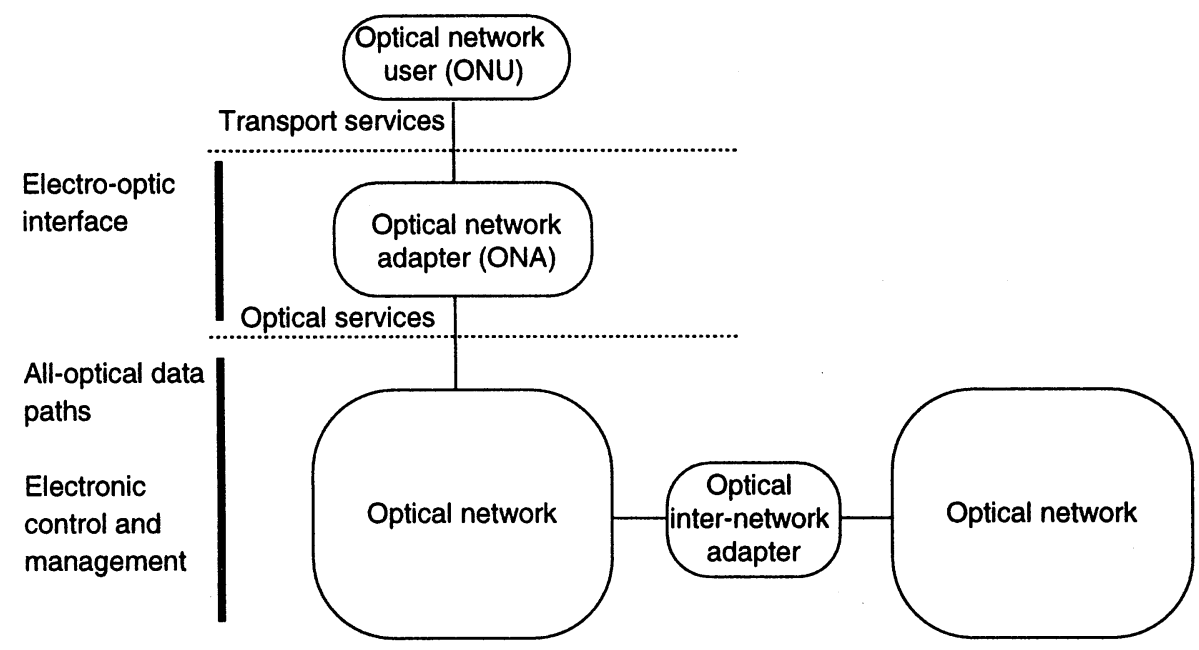

taken from "Optical Services in Future Broadband Networks" by Finn \& Barry, IEEE Network, Nov/Dec 1996

Fig. 2-1 Optical network boundary

This paper shows how the layered network architecture model maps into this representation of the optical network. It also describes some of the considerations related to the layered architecture model such as the characteristic information, the client/server relationship between the adjacent layer networks and the overhead information.

\section{Optical transport network layered architecture}

The layering concept as defined in G.805 is applied to the optical WDM transport network in [2], [3] and [4] which resulted in several architecture models. All models, though slightly different introduce three new optical layer networks. These three new layers fully represent the management 
capabilities offered in WDM optical transport network and should be sufficient for the purposes of TMN and network resources specification also in the project MOON. The architecture model currently used in the MOON resembles the G.otn model extending some aspects which are currently not covered. It is also one of the project objectives to take the G.otn model under consideration and to give comments according to the experiences gained in the project.

The model proposed in ITU-G.otn define three layer networks :

- optical channel layer,

- optical multiplex section layer and

- optical transmission section layer.

Most of the other models propose the term optical path layer instead of term optical channel layer. In the MOON the term "optical path „ is preferred to the term "optical channel" because it better describes end-to-end networking of the client signal and has no physical connotations.

The layered architecture model for the optical transport network :

- supports modelling of different optical equipment in the network,

- should fit into the existing layering scheme defined for the other transport technologies but should not be restricted only to them. In this way the simple modelling of networks that contains multiple transport technologies can be provided.

- encapsulate different transport and management capabilities in different layer networks. The optical path layer network support end-to-end networking of an arbitrary single optical signal. The multiplex section network support end-to-end networking of a multi-wavelength optical signal. The transmission section network support integrity of transmission on the optical media -fibre. Each layer network is characterised by its characteristic information.

In the Fig.3-1 the layer architecture model of the ITU-G.otn is used to describe the interconnection between optical path network user and optical path network. The models of the optical terminal multiplexer and the optical cross-connect are also shown. 
The client network depicted in Fig.3-1 is the representation of the arbitrary network which can use services offered by the optical path layer network. The optical network adapter offers the transparent access to the networking capabilities of the optical network, and transforms the arbitrary user characteristic information into the optical signal. Due to this function, the optical path network adapter is placed at the boundary of the optical path network. The interfaces between the user, adapter and network can be physical $\left({ }^{*} 2\right)$, $\left({ }^{\star} 3\right),\left({ }^{*} 5\right)$ or logical $\left(1^{*}\right),\left({ }^{*} 4\right)$. The existence of the logical interface implies that the two interconnected parts could be physically integrated. In the Fig. 3-1 some possible types of transport interconnections are shown.

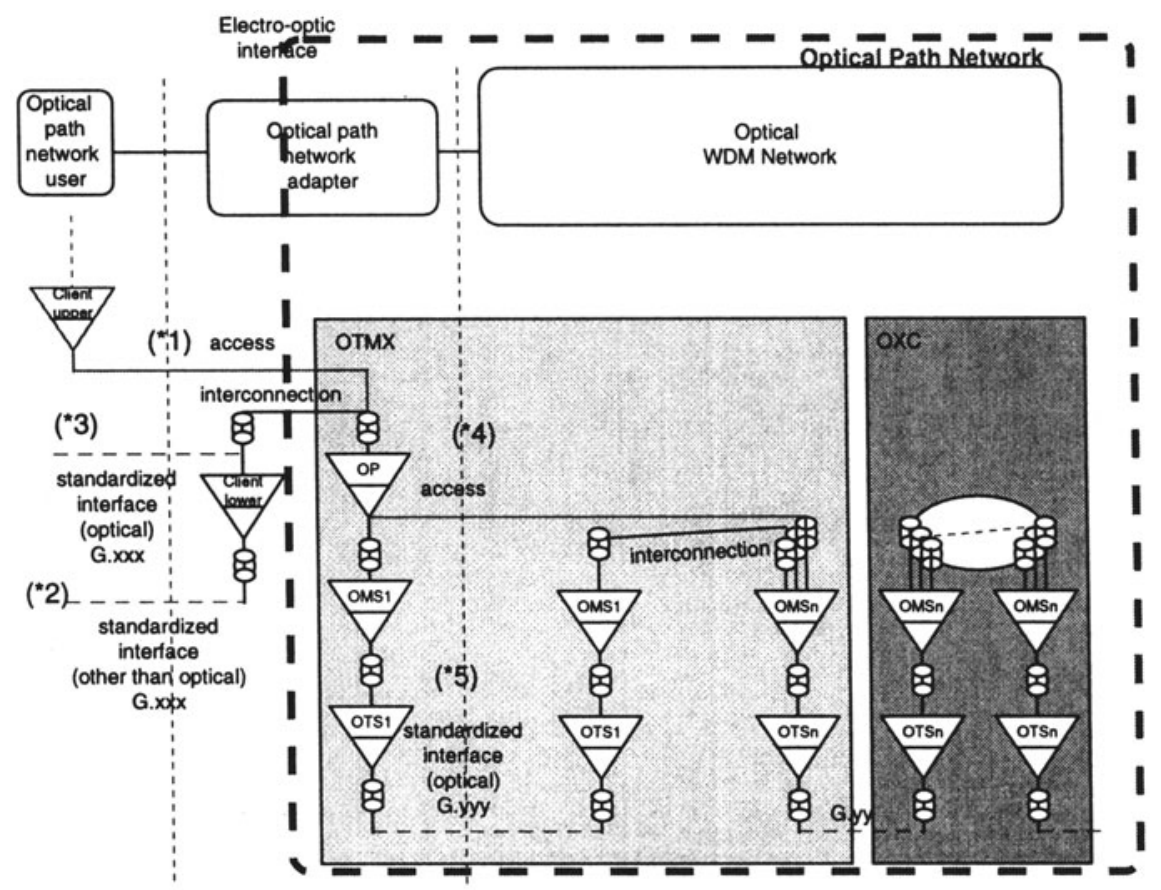

Fig. 3-1 Optical Path Network

The interface at the boundary of the optical path network supports the input characteristic information of the optical path layer network. The optical path layer network could in principle provide both optical and logical interface. 
This means that the input characteristic information of the optical path layer can also have a physical representation. In the Fig. 3-1 the logical interface (*1) and the physical optical interface (*2) are depicted.

The interface $\left({ }^{*} 5\right)$ is the physical interface between optical network adapter and optical network. It represents ITU-G.otn optical interface. The optical signal on this interface has the characteristic information of the optical transmission layer network and supports overhead and supervisory channels defined for all layer networks.

The interface (*4) depicts the logical interface between the optical network adapter and the optical network. In this case the adapter is integrated in the optical network equipment.

The architecture model of the client network comprises two layer networks, upper layer and lower layer. The lower layer of the client network is a kind of a physical media layer. We assume that between two client layer networks the logical interface exists - the output characteristic information of the upper layer has no physical representation.

The optical path layer network can be a server for this client layer network if the output characteristic information of the client layer is defined in the same way as the input characteristic information of the optical path layer network. In this case in the layering scheme the optical layer networks can be embedded under the client upper layer network. The ITU-G.otn model defines only the logical interface for the optical path layer network. Some other models propose that also the physical interface - single wavelength optical signal - should be supported. The interface (*3) depicts this case.

In this contexts the definition of the characteristic information, the overhead information and the client/server relationship for each layer network is the first important task. 


\subsection{Characteristic Information of the Optical Layer Networks}

In the project MOON the definition of the characteristic information of the layer networks is adopted from the architecture models defined in [2] and in [4].

The characteristic information of an optical path layer network is a single wavelength optical signal with the optical frequency in defined wavelength range, power level in defined power level range, modulation bandwidth in defined bandwidth range. The signal is conditioned for the all-optical transport and supplemented by the optical path overhead information.

The characteristic information of an optical multiplex section layer network is an optical multi-wavelength signal with defined WDM grid, defined power level range, the restriction of group velocity dispersion, supplemented by an optical multiplex section layer overhead information.

The characteristic information of an optical transmission section layer network is an optical multi-wavelength signal of defined optical signal parameters such as power level, SNR, etc. supplemented by the overhead information.

The characteristic information of a layer network describes a resource which can be used for a transport of a client information. It should also define a resource for a transport of the overhead information of this layer network.

3.2 Overhead information and the concept of the supervision network

The overhead information definition represents a part of the "specific protocol" defined for one layer network, which guarantees the integrity of the transport of the client characteristic information. One part of the overhead information is the trail overhead information.

The trail overhead information is "added" to the adapted client characteristic in the trail termination source and "extracted" at the trail termination information sink. So according to this definition the overhead information is a part of the trail which is assigned to a single network connection. 
This means that in the optical path layer network the optical path trail overhead must be transported embedded. The non-embedded overhead information - the overhead information carried by another optical channel is assigned to the different network connection, hence it does not belongs to the same trail. The optical multiplex section layer trail overhead information must be carried by a channel which belongs to the defined WDM grid. For the optical transmission section trail overhead information a channel which does not belongs the WDM grid can be utilised. The trail overhead information of one layer network is created, processed and terminated in that layer.

In the optical network the concept of the supervision network for transport of the trail overhead information of the multiplex and transmission layer network as well as the overhead information which is not related to the trail, such as OAM data used for the alarm suppressing purposes (inter-level alarming) and for the control of the automatic OAM actions e.g. automatic protection switching, can be introduced. The supervision network can be a standardised part of the transport optical network architecture as proposed in ITU-G.otn. In this case the optical transmission layer provides access point for the supervision information of all layers. This information has a logical interpretation in its containing layer and becomes its physical interpretation in the optical transmission layer which provides network connection for a supervision network. The establishment and monitoring of the supervision network connection is also performed in the optical transmission layer.

The standardised layered architecture model for the optical network should be applicable for the modelling of the arbitrary optical WDM network. Still, when it comprises also the definition of the overhead channels or the supervisory network it puts restrictions on the modelling of some optical equipment. Some very simple optical equipment can not be modelled with this, because it supports no termination points of the supervision network where supervision data could be accessed.

Hence, it could be assumed that in the future some parts of the network will not support the whole standardised functionality, the transport of the supervision data in the first place, but can still be used for the transport. The layer network in which the supervision network connection is established and monitored has to be able to deal with this exceptional situation. 


\subsection{Client/Server Relationship}

The transport of the client information is the "service" offered by a layer network which is supported and guaranteed by a "protocol " specific for this layer network.

The service defines how the characteristic information of the client network can be adapted and enhanced to form the characteristic information of the server network. This comprises the definition of transport processing functions: adaptation and termination function and the definition of the overhead information.

\section{The protocol comprises}

- the definition of the two counter-part management processes in the same layer and the information they exchange. This can be related to the definition of the termination function. The information exchanged cab is a part of the layer network trail overhead information, and can be transported in the layer network overhead.

- the definition of management processes in two adjacent layers and the information they exchange. This can be related to the definition of the adaptation function. The adaptation function has access to the original characteristic information of the client, which means that it can also have an access to the client overhead information.

\subsubsection{Adaptation function}

The adaptation function is a "transport processing function" which adapts client to the requirement and limitation of the server layer. It defines the "server/client" association between the client layer connection point and server layer access point, which delimit the adaptation function.

The transport related functions of the adaptation function for all three layer networks are defined in the ITU-G.otn and should be only enhanced for the case when the optical path layer network also excepts an optical signal.

The management related functions of the adaptation function can be described as follows: 
- The adaptation sink performs monitoring of the client connections when a multiple of client connections are transported over a single trail in server network. This is the case in the optical multiplex section layer and it includes loss of channel, charier wavelength shift, degradation of the signal quality.

- The adaptation sink generates supervision information ( OAM data such AIS, etc. ) to be sent to the client layer network trail termination sink.

\subsubsection{Trail termination function}

The trail termination function is a "transport processing function" which generates the characteristic information of the layer network and ensures integrity of that characteristic information. The trail termination defines the association between the access point and the termination connection point, which delimit the trail termination function.

The transport related functions of the trail termination function can be described as follows.

- The trail termination source accepts adapted client characteristic information, creates trail overhead and assigns it to an associated network connection.

- The trail termination sink terminates the trail, terminates the trail overhead information and passes the adapted client characteristic information to the adaptation function.

The management related functions are described as follows:

- The trail termination source generates supervision information ( OAM data such as remote defect indication in all layer networks or shift of frequency degradation in the optical multiplex layer network, etc. ) to be sent to its counter-part trail termination sink

- The trail termination sink performs validation of the trail overhead,

- The trail termination sink performs assessment of transmission quality of the characteristic information, fault detection and alarming, 
- The trail termination sink process supervision information received from its counter part or a server layer network (when monitoring of the connections is done in the server layer network).

\section{Layer Network Management Processes}

The management process in one layer network is concerned (1) with the normal operation of the resources of layer network including automatic processes such as resource status monitoring, protection switching, alarm detection ,automatic alarm suppressing, fault localisation and (2) it should support the interface to the TMN management system.

The management processes in different layer networks can communicate in several different ways. The client layer network management process can supply its management information to the server layer network management process, or to its distinct counter-part

- as part of its own characteristic information

- as an information carried by the supervisory network

- directly, when the processes are collocated in the single agent

Two management process can also communicate only with a management system, and not with each other. In this case their relationship and correlation of the supplied information are managed by the manager.

The access point represents the boundary between two different layer networks where the client information enters and leaves the server network. Between two network access points the connection in the server network is established and two different management processes are related to it :

- the management process in the client network which requires the establishment of the connection, relates two access points and provides the unique identification of the client to the server

- the supportive management process in the server network. This process performs monitoring of the connection integrity by a means of trail monitoring or connection monitoring, alarm detection, etc.

Here we will only discuss connection monitoring.

As stated in [2] the integrity of the information transferred over connection can be monitored using one of the techniques: (1) inherent monitoring , using the data inherently available from the trail in the server layer network, 
(2) non-intrusive monitoring, by use of listen only monitoring of the original client characteristic information, (3) intrusive monitoring, by breaking the original trail and introducing a test trail that extends over the part of the connection of interest for the duration of the test, and (4) sublayer monitoring, by a trail created in a sublayer.

For the monitoring of the optical client network connection and optical multiplex section connections the inherent monitoring can be applied.

Since the adaptation function in the optical multiplex section layer includes multiplexing the error performance of each link connection in an optical path layer supported by a trail will not be available individually, so for the monitoring of the optical path connections the non-intrusive monitoring must be applied. The listen only monitoring of the characteristic information of the optical path layer network is performed in the optical multiplex section layer connection point, and includes:

- monitoring of the demultiplexed WDM channels

- non-intrusively reading of the optical path overhead information related to monitored channels in order to relate the defects detected for the WDM channels to the unique optical path identifier.

In the definition of the management information model the part of the layer network management process which is concerned with the trail monitoring (inherent characteristic information monitoring) is assigned to the managed object termination connection point of the layer network.

The non intrusive connection monitoring is assigned to the managed object connection termination point between client and server layer where the related connections are accessible before they are being multiplexed in the server layer.

Other aspects, such as protection switching, etc. are also covered by the management information model of the project MOON and will be demonstrated in MOONET.

\section{Layer Network Resources}

In the sense of the network management G.805 architectural components represent network resources. Since they are defined in general way in [2], in the standard I-ETS 300-653 [5] the attempt was made to define, in the general way, also the related managed objects and management capabilities. 
The topological components describe network resources in the domain of the static configuration resource management. Static resource management is mainly concerned with the provisioning of the resources such as the provisioning of a layer network and characteristic information, the provisioning of access points, the provisioning of access groups, the configuration of access groups, the provisioning and the configuration of connection points, the provisioning and configuration of subnetworks and links.

The transport entities describe network resources in the domain of the dynamic configuration connection management that is concerned with the set-up, modification and release of connections such as sub-network connection set-up, the release of sub-network connections, sub-network configuration, scheduling, trail set-up and release, the setting-up of network connections, which comprises: the configuration of the link, the provisioning of link connections and tandem connection provisioning and configuration, and the release of network connections.

The Figure 5-1 depicts the whole set of the G.805 architectural components.

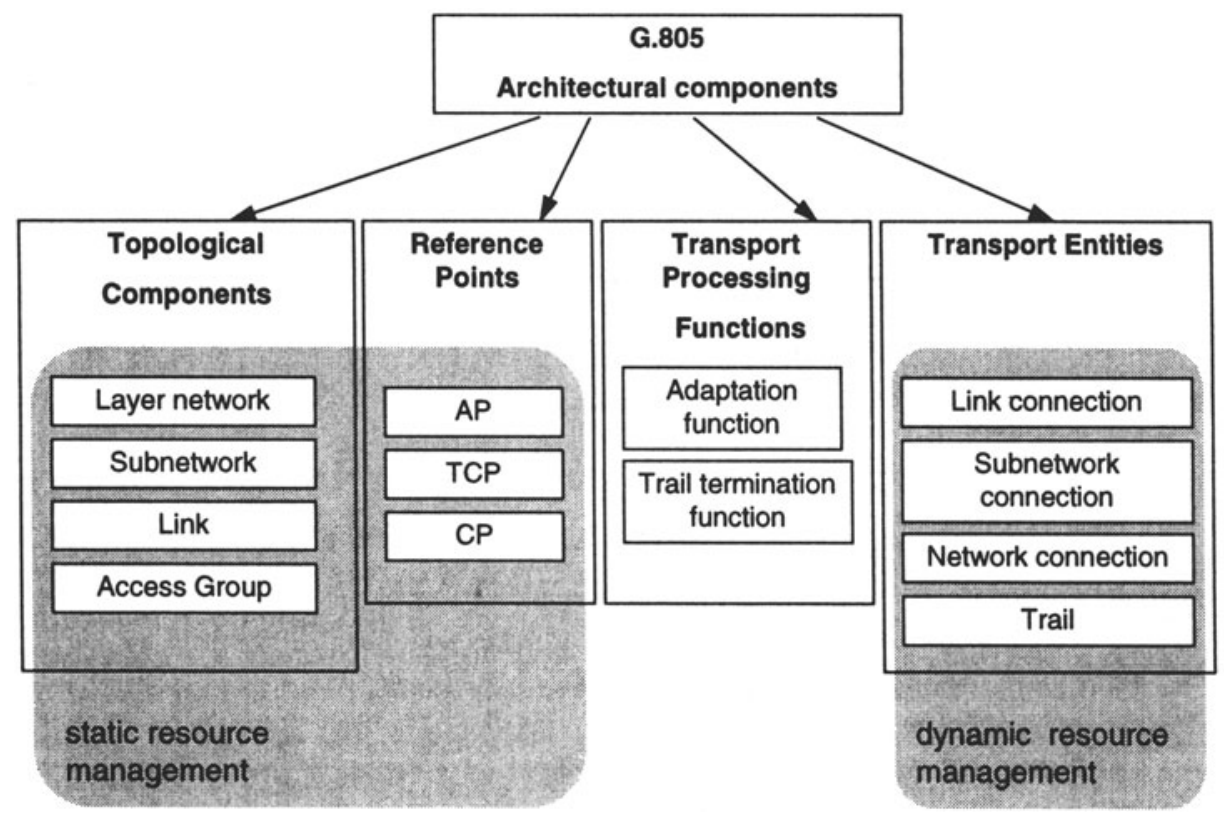

Fig. 5-1 G.805 Architectural components 
In the project MOON the approach similar to the approach utilised in [5] is taken for the definition of the management information model. This comprises the utilisation of the adopted layered network architecture in modelling of the network elements and all-optical equipment, the identification of the network resources, and the definition of the related management capabilities.

Within one layer network all resources can be described with the architectural components defined within this layer. In the table in Fig. 5-2 the components that can be considered to represent network resources in different optical transport layer networks are depicted. The symbol (*) depicts that this component is used to describe the resources of the MOONET.

\begin{tabular}{|l|l|l|l|l|l|l|l|}
\hline \multirow{2}{*}{$\begin{array}{l}\text { Topological } \\
\text { component }\end{array}$} & \multicolumn{2}{|l|}{$\begin{array}{l}\text { Defined for a } \\
\text { layer network }\end{array}$} & \multicolumn{2}{l|}{$\begin{array}{l}\text { lransport } \\
\text { entity }\end{array}$} & \multicolumn{3}{|l|}{$\begin{array}{l}\text { Defined for a } \\
\text { layer network }\end{array}$} \\
\cline { 2 - 7 } & OP & OMS & OTS & & OP & OMS & OTS \\
\hline Layer network & $X\left(^{*}\right)$ & $X\left(^{*}\right)$ & $X\left(^{*}\right)$ & $\begin{array}{l}\text { Link con- } \\
\text { nection }\end{array}$ & $X\left(^{*}\right)$ & $X\left(^{*}\right)$ & $X\left(^{*}\right)$ \\
\hline Sub-network & $X\left(^{*}\right)$ & $X$ & - & $\begin{array}{l}\text { Sub-network } \\
\text { connection }\end{array}$ & $X\left(^{*}\right)$ & $X$ & - \\
\hline Link & $X$ & $X$ & $X\left(^{*}\right)$ & $\begin{array}{l}\text { Network } \\
\text { connection }\end{array}$ & $X$ & $X$ & $X$ \\
\hline Access group & $X\left(^{*}\right)$ & - & - & Trail & $X\left(^{*}\right)$ & $X\left(^{*}\right)$ & $X\left(^{*}\right)$ \\
\hline Access point & $X\left(^{*}\right)$ & $X\left(^{*}\right)$ & $X\left(^{*}\right)$ & & & & \\
\hline Connection point & $X\left(^{*}\right)$ & $X\left(^{*}\right)$ & $X\left(^{*}\right)$ & & & & \\
\hline
\end{tabular}

Fig. 5-2 Network resources

The subnetwork and subnetwork connection are defined for both optical path and optical multiplex section layer. This represents both end-to-end networking of the client signal and WDM signal. The access group is defined only for optical path layer where collocated optical signals are multiplexed in the same link (same fibre).

The emerging management information model would be demonstrated in the field trial network MOONET. Due to the relatively simple topology of the MOONET not all of the concepts established in MOON could be verified. 
Still, the activities in the project MOON are aimed to give the contribution to the general view of the optical network modelling and management.

\section{Conclusions}

The layered architecture model covers all the technology aspects of the optical transport network. It can be used for equipment modelling, network access modelling, and the network resource modelling. For each layer network a managed process which interacts with its counter-part in the same layer or with the adjacent layer management processes can be defined. This process can be partly assigned to the adaptation and termination functions of the layer network. It must also provide an interface to the TMN agent. In this way the interaction of the OAM and the TMN can be modelled. The interaction between two different technology transport networks can be modelled with the interaction between management processes of two adjacent layer networks which belong to different technology transport networks. In the project MOON the optical transport network layered architecture is used as the starting point for the definition of the TMN resources, and of the related TMN management information model.

\section{Acknowledgement}

The optical transport network layered architecture is a topic of main a interest for a number of partners participating in the project MOON. It has been recognised that the layered architecture model should be the basis for the management considerations, so many discussions and consultations started, and are still in progress. The author, who is actively participating in the team responsible for the definition of the information model for the optical network management in the project MOON, took also part in this discussions and contributed the project intern workshop which aimed to enlighten this topic from many different points and to tackle many different aspects such as the necessity to establish a model able to represent also network elements such as $2 \mathrm{R}$ and $3 \mathrm{R}$ regenerators, different client interconnections etc. This paper evolved from the contribution of the management information model team to this workshop. The author would like to mention all MOON partners who are currently engaged and contributing to this topic : CSELT Italy, DT Germany, Siemens Germany and Siemens Austria. 
8. References

[1] ITU-T G.805 „Generic Functional Architecture of Transport Networks“

[2] ITU-T G.otn „Architecture of Optical Transport Networks“

[3] ITU-T G.681 „Functional Characteristics of Interoffice and Long-Haul Line System Using Optical Amplifiers, Including Optical Multiplexers“

[4] Mathias Bischoff: „Formal Description of Optical Transport Networks“

[5] ETSI I-ETS 300653 TMN; "TMN Generic Managed Object Class Library for the Network Level View";

[6] Ken-ichi Sato„Photonic Transport Network OAM Technologies“, IEEE Comm. Mag. Dec. 1996.

[7] Steven G. Finn at al. „Optical Services in Future Broadband Networks“ , IEEE Network Nov/Dec 1996

[8] Mathias Bischoff at al. „Operation and Maintenance for an All-Optical Transport Network", IEEE Comm. Mag. Nov. 1996. 